\title{
Application of Spectral Information to Investigate Historical Materials \\ - Detection of Metameric Color Area in Icon Images -
}

\author{
Kimiyoshi Miyata ${ }^{1}$, Hannu Laamanen ${ }^{2}$, Timo Jaaskelainen ${ }^{2}$, \\ Markku Hauta-Kasari ${ }^{3}$, and Jussi Parkkinen ${ }^{3}$ \\ 1 Museum Science Division, Research Department, \\ The National Museum of Japanese History, \\ 117, Jonai-cho, Sakura-shi, Chiba 285-8502, Japan \\ miyata@rekihaku.ac.jp \\ http: / / www.rekihaku.ac.jp \\ ${ }^{2}$ Color Research Group, Department of Physics, University of Joensuu, \\ P.O. Box 111, 80110 Joensuu, Finland \\ \{hannu.laamanen, Timo.Jaaskelainen\}@joensuu.fi \\ http://spectral.joensuu.fi/ \\ ${ }^{3}$ Color Research Group, Department of Computer Science, University of Joensuu, \\ P.O. Box 111, 80110 Joensuu, Finland \\ \{mhk, jussi.parkkinen\}@Cs.joensuu.fi \\ http://spectral.joensuu.fi/
}

\begin{abstract}
The spectral reflectance of Icons is estimated from RGB digital images taken by a digital camera, and it is applied to detect metameric color areas in the Icons. In this paper, two detection methods are proposed and examined by using a test chart and ten Icons painted on wooden plates. The first method is based on the definition of metamerism that two stimuli can match in color while having a disparate spectral reflectance. The second method is based on a phenomenon that the variation of the color difference between two colors is changed by replacing the illuminant if the colors are metamers to each other. The experimental results can be used to consider which parts of the Icons have been repainted as restoration treatments. Despite the necessity of further consideration and improvement, the experimental results demonstrate that the proposed methods have the basic ability to detect metameric color areas.
\end{abstract}

\section{Introduction}

Historical materials tend to be very fragile, and thus, they need to be preserved from further degradations in future. Photography of materials is one of the tools to achieve both conservation and utilization of the objects. However, information recorded in photographs is not sufficient to analyze the materials for historical research and advanced investigation purposes since they have only three color channels, which have device dependency. Spectral reflectance has a variety of objective and deviceindependent information on the materials. In fact, many researches have been con- 
ducted on the measurement and estimation of spectral reflectance, and its application to various fields of study. Not only does spectral reflectance encourage the investigation of materials, but it also forecasts restoration treatments and natural fading processes [1]-[3]. It also provides opportunities to produce precise reproductions with highly accurate color for various lighting conditions. This study introduces methods with the use of spectral reflectance to investigate authentic historical artifacts.

In this paper, spectral reflectance is used to detect metameric color areas in the Icons. Metamerism is a well known phenomenon of color that is defined that metameric color stimuli are color stimuli with the same tristimulus values but different spectral radiant power distributions [4]. In the conservation and restoration of historical materials, evidence on restoration treatments, which have been applied to the objects, is important information. In such restoration treatments, color dyes or pigments having metamerism relation to the original pigments would be used. Thus, metameric color area could tell us which parts of an object have been possibility repainted. In this study, it is examined by using color and spectral information on authentic Icons. Two detection methods will be proposed and their details will be described in the following sections.

\section{Obtaining Spectral Reflectance of Historical Materials}

There is a variety of techniques to obtain the spectral reflectance of objects that can be classified primarily into two categories - the measurement techniques with the use of measuring equipments and the estimation techniques based on some mathematical models. One of the measurement techniques was presented in our previous study [5], while an estimation technique is applied in this study.

\subsection{Estimation of Spectral Reflectance}

Many recent studies have been addressing the estimation of spectral reflectance for a variety of materials [6], [7]. However, there are problems with the measurement of spectral reflectance, including the long measurement time, sensitivity of the photo detector, low spatial resolution compared to recent digital cameras, and large quantity of measured spectral data. In order to overcome these issues, the Wiener estimation technique is introduced to obtain the spectral reflectance of materials from images taken by a digital camera having RGB color channels. In this study, this technique is applied to estimate the spectral reflectance of Icon images. The Wiener estimation matrix $\mathrm{M}$ is determined as follows [8]:

$$
\begin{gathered}
\boldsymbol{v}=\left[d_{R}, d_{G}, d_{B}, d_{R}^{2}, d_{G}^{2}, d_{B}^{2}, d_{G} d_{B}, d_{R} d_{B}, d_{R} d_{G}, d_{R} d_{G} d_{B}\right]^{t} \\
\mathrm{R}_{r v}=<\boldsymbol{r} \boldsymbol{v}^{\mathrm{t}}> \\
\mathrm{R}_{v v}=<\boldsymbol{v} \boldsymbol{v}^{\mathrm{t}}> \\
\mathrm{M}=\mathrm{R}_{r v} \mathrm{R}_{v v^{-1}}^{-1}
\end{gathered}
$$


where vector $\boldsymbol{r}$ is the measured spectral reflectance of the Macbeth Color Checker and vector $\boldsymbol{v}$ consists of the sensor response $d_{R}, d_{G}$ and $d_{B}$ including higher order terms when the Checker is taken as a digital image. Matrix $\mathrm{R}_{r v}$ is a crosscorrelation matrix between vector $\boldsymbol{r}$ and $\boldsymbol{v}$, while Matrix $\mathrm{R}_{v \boldsymbol{v}}$ is an auto-correlation matrix of vector $\boldsymbol{v}$. The symbol $<\cdot>$ represents the ensemble average, and $\mathrm{t}$ is the transpose of the vector. Spectral image data $f(x, y, \lambda)$ is calculated from vector $v$ and the matrix $\mathrm{M}$ as:

$$
f(x, y, \lambda)=\mathrm{Mv}
$$

The Icons used in the estimation of spectral reflectance are shown in Figure 1. Natural pigments have been applied on wooden plates of these mid-19 $9^{\text {th }}$ century Icons. The minimum size of the Icon is approximately $240 \mathrm{~mm}$ in height and $165 \mathrm{~mm}$ in width, while the maximum size is about $430 \mathrm{~mm}$ in height and $330 \mathrm{~mm}$ in width. The images of these Icons are taken by a consumer-type digital camera.

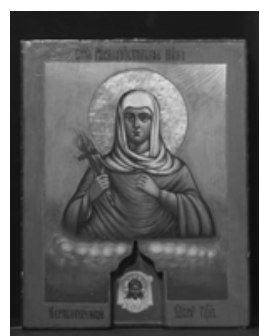

(a) Icon No.1

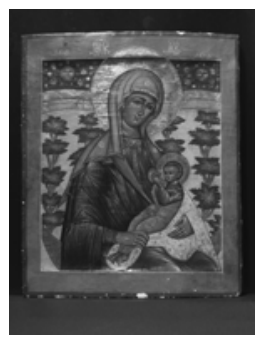

(d) Icon No.4

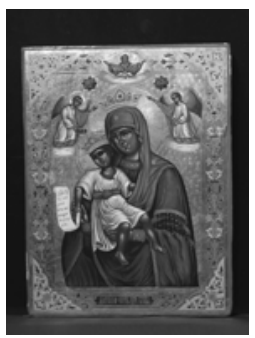

(g) Icon No.8

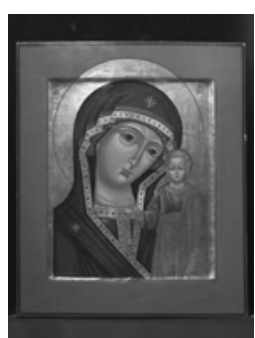

(b) Icon No.2

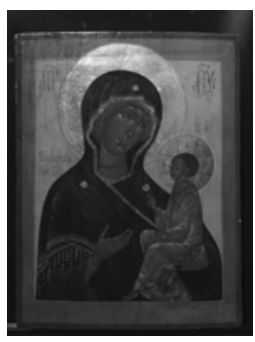

(e) Icon No.6

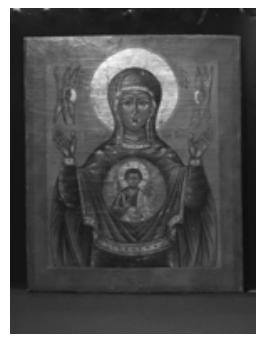

(h) Icon No.9

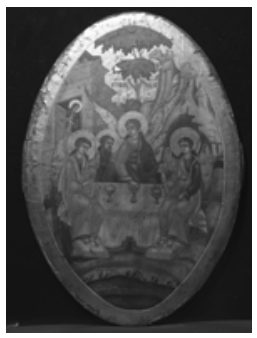

(i) Icon No.10

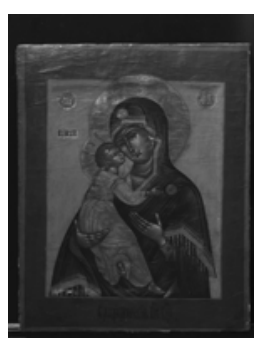

(c) Icon No.3

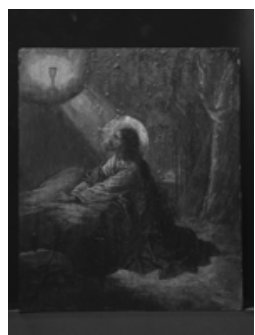

(f) Icon No.7

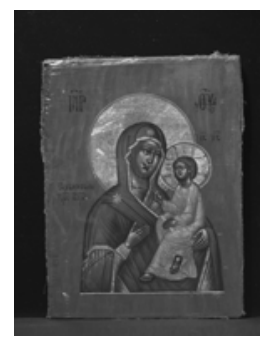

(j) Icon No.18

Fig. 1. Icons used in the study. These RGB images are taken by a digital camera 


\subsection{Applications of Spectral Reflectance}

The estimated image of spectral reflectance for each Icon can be used for a wide range of applications. For example, Figure 2 shows spectral color separation, which

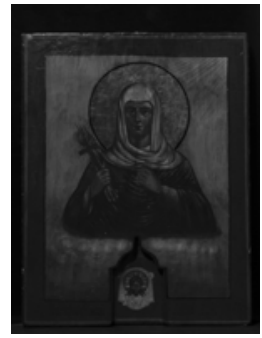

(a) $400 \mathrm{~nm}$ channel

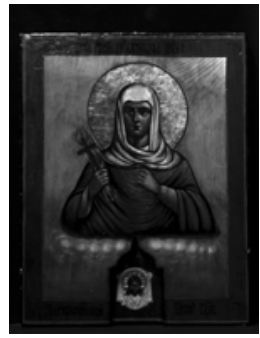

(b) $500 \mathrm{~nm}$ channel

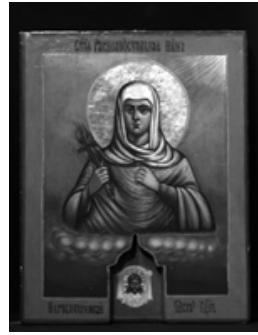

(c) $600 \mathrm{~nm}$ channel

Fig. 2. Spectral channel separation using the estimated spectral reflectance

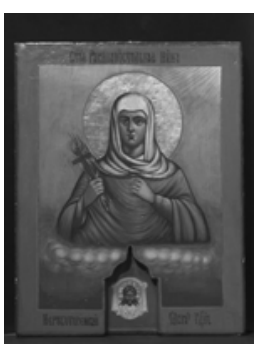

(a) under D65

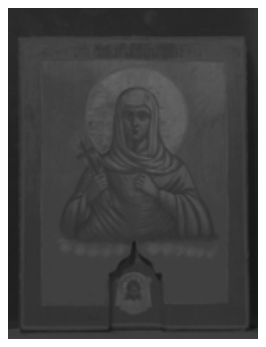

(b) under V1

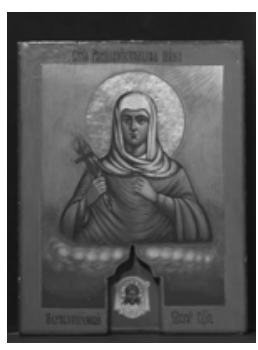

(c) under V2

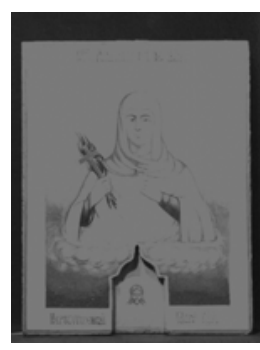

(d) under V3

Fig. 3. Simulation of color changes under different illuminants shown in Figure 4

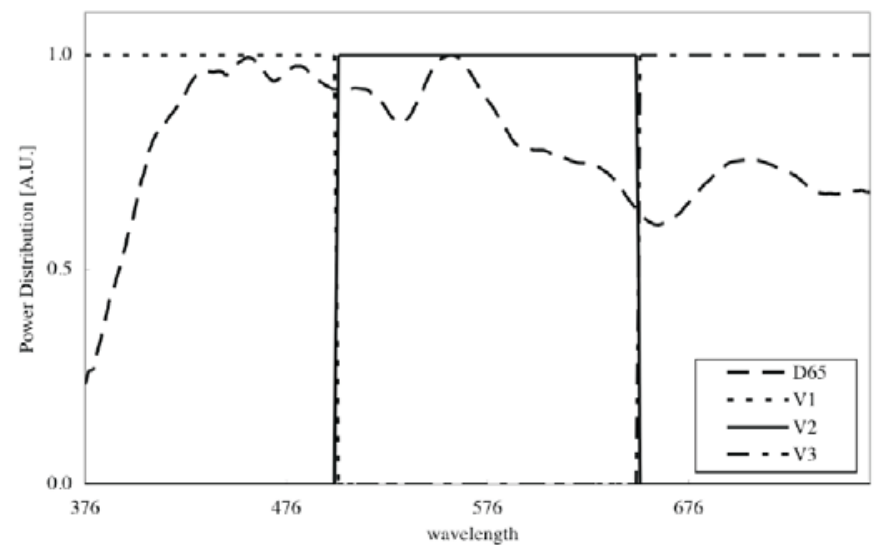

Fig. 4. Spectral power distributions of the D65 and three virtual illuminants, V1, V2 and V3 
helps consider the types of pigments used to paint the Icons. Figure 3 illustrates the simulation of color changes under different illuminants. This simulation is useful to test the color changes of a material under various illuminants, such as those in galleries or research laboratories.

In Figure 3, (a) is a RGB image calculated from the estimated spectral reflectance image under the D65 illuminant, and (b), (c), and (d) are simulated color reproductions under three different kinds of virtual illuminants. The spectral power distributions of virtual illuminants are rectangular in shape that corresponds to the domain of short, middle, and long wavelengths as shown in Figure 4. These virtual illuminants are also used in the experiment to detect the metameric color areas described in the next section.

\section{Detection of Metameric Color Area}

\subsection{Purpose to Detect Metameric Color Area}

In the investigation of historical materials, the records of restoration treatments applied to an artifact provide valuable information on how the object has been restored. This information contributes to historical researches, and further renovation and conservation of the object. Restoration records could be found by scientific or chemical analysis. However, these analyses often require fragments removed from the materials. One of the ideas to detect restoration records without any destruction to the objects is the use of color and spectral information. When historical materials were restored in the past, color dyes or pigments used in the restoration would be selected to be observed the same color to the original materials, but not identical in the sense of spectral reflectance. This is matched to the definition of the metamerism. Thus, the detection of metameric color areas in an artifact suggests which parts of the object have been restored.

In this article, two methods to detect metameric color area are proposed and applied to the Icons. The first method is based on the definition of metamerism that two stimuli can match in color while having disparate spectral reflectance. The second methods is based on a phenomenon that the variation of color difference between two colors will be changed if a different illuminant is used and the colors are metamers each other. The details of the detection methods will be described in the following sections.

\subsection{Proposed Method}

\subsubsection{Method 1}

The metameric color area has the same tristimulus value but different spectral reflectance. The first detection method is based on this definition of metamerism. In Method 1, the color information is evaluated by using $\Delta E$ and spectral information is 
evaluated by using the RMSE (root mean square error). The $\triangle E$ and $R M S E$ are calculated by the following equations:

$$
\begin{gathered}
\Delta E(x, y)=\sqrt{\left\{L^{*}\left(x_{r}, y_{r}\right)-L^{*}(x, y)\right\}^{2}+\left\{a^{*}\left(x_{r}, y_{r}\right)-a^{*}(x, y)\right\}^{2}+\left\{b^{*}\left(x_{r}, y_{r}\right)-b^{*}(x, y)\right\}^{2}} \\
\operatorname{RMSE}(x, y)=\frac{1}{N_{\lambda}} \sqrt{N_{j=1}^{N_{\lambda}}\left\{f\left(x_{r}, y_{r}, \lambda_{j}\right)-f\left(x, y, \lambda_{j}\right)\right\}^{2}}
\end{gathered}
$$

In the equations, $f(x, y, \lambda)$ is the estimated spectral reflectance image, $N_{\lambda}$ is the number of spectral dimension, $(x, y)$ is the pixel position, and $\left(x_{r}, y_{r}\right)$ is the reference pixel position. $L^{*}(x, y), a^{*}(x, y)$, and $b^{*}(x, y)$ correspond to CIE $L^{*} a^{*} b^{*}$. First in Method 1, we select an arbitrary pixel position in the Icon image. The selected pixel is referred to the reference pixel to detect the metameric color. The procedure of Method 1 is listed as follows:

Step 1: Set the position of the reference pixel.

Step 2: Observe the spectral reflectance at the pixel position, and use it as the reference spectral reflectance.

Step 3: Check every pixel in the image whether the color difference between the current pixel and reference pixel is less than a predetermined threshold $T_{c}$.

Step 4: Check every pixel in the image whether the RMSE between the current pixel and reference pixel is greater than a predetermined threshold $T_{R M S E}$.

Step 5: Mark the pixels that satisfy both Step 4 and Step5 as detected metameric color.

\subsubsection{Method 2}

We proposed another detection method as Method 2. As in Method 1, we first select an arbitrary pixel position in the Icon image that is referred to the reference pixel. Four kinds of illuminants shown in Figure 4 are used, and $\Delta E_{i}(i=1,2,3$ and 4$)$ under the each illuminant $i$ is calculated from Equation (8). The difference between color differences calculated from two illuminants by using Equation (9). One of them is the color difference calculated for D65 illuminant as the reference illuminant $(i=1)$ in this study. The difference is compared with thresholds to determine whether the current pixel is metameric color to the reference pixel.

$$
\begin{gathered}
\Delta E_{i}(x, y)=\sqrt{\left\{L_{i}^{*}\left(x_{r}, y_{r}\right)-L_{i}^{*}(x, y)\right\}^{2}+\left\{a_{i}^{*}\left(x_{r}, y_{r}\right)-a_{i}^{*}(x, y)\right\}^{2}+\left\{b_{i}^{*}\left(x_{r}, y_{r}\right)-b_{i}^{*}(x, y)\right\}^{2}} \\
\left|\Delta E_{1}(x, y)-\Delta E_{2}(x, y)\right|>T_{2} \\
\left|\Delta E_{1}(x, y)-\Delta E_{3}(x, y)\right|>T_{3} \\
\left|\Delta E_{1}(x, y)-\Delta E_{4}(x, y)\right|>T_{4}
\end{gathered}
$$

The metameric color areas have to be in small color difference. This is evaluated by using $\Delta E$. Three virtual illuminants are used to emphasize the change of 
the color difference cause of metamerism effect because the color difference between the current and reference pixel is kept if the both pixels are in metamerism relation. All of pixels in the image are checked based on the sequence listed as follows:

Step 1: Set the position of a reference pixel.

Step 2: Calculate $\Delta E_{i}$ for four kinds of illuminants from Equation (8).

Step 3: Select pixels with $\triangle E_{1}$ less than predetermined threshold $T_{1}$ as metameric color candidates.

Step 4: Check $\Delta E_{2}, \Delta E_{3}$, and $\Delta E_{4}$ for all of the candidates in Step 3.

Step 5: Select pixels satisfying any one of the Equations (9).

Step 6: Mark the pixels that satisfy both Step 3 and Step 5.

\subsection{Experimental Results}

First, Method 1 is conducted with the use of a test chart, which has metamerism color patches, as shown in Figure 5. In the figure, (a) shows the original chart, and (b) and (c) show the detected area by using only $\triangle E$ and by using RMSE to the reference pixel respectively. The white dot in the figure is the predetermined reference pixel, and the gray area is a collection of pixels detected as metameric color area to the reference pixel. Figure 5 (d) shows the final result of the detected metameric color area to the reference pixel that is determined by both $\triangle E$ and RMSE. Method 1 is also applied to the authentic Icon images, and the results are shown in Figure 6. Each Icon in the figure has a single reference pixel, which can be set at an arbitrary position in the image.

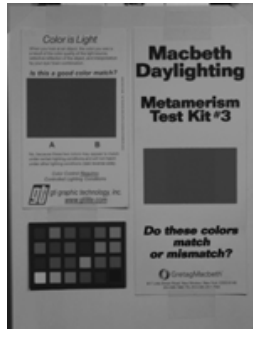

(a) Original chart

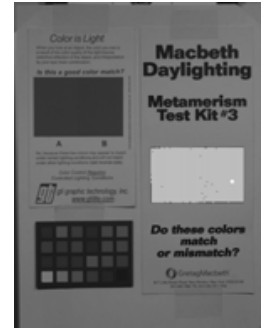

(b) Detected pixels $(\Delta E<10.0)$

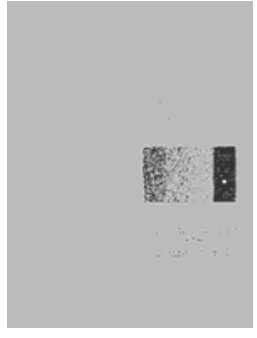

(c) Detected pixels (RMSE > 0.04)

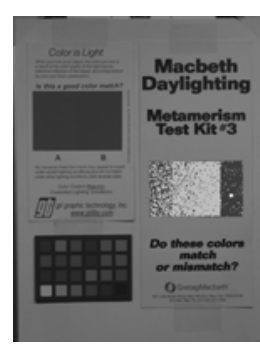

(d) Detected pixels by both $\triangle E$ and RMSE

Fig. 5. Results of Method 1 by using a metamerism test chart

Subsequently, Method 2 is applied to the test chart and Icon images. Figure 7 (a) shows the original chart while (b) shows the detected metameric color area. The results of the experiment on Icon images are shown in Figure 8. 


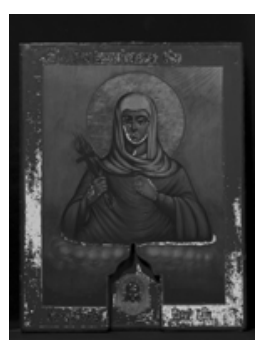

(a) Icon No.1

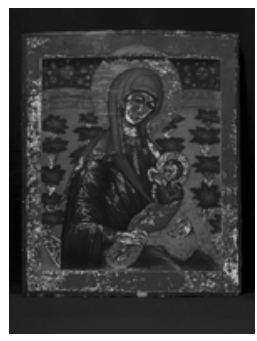

(d) Icon No.4

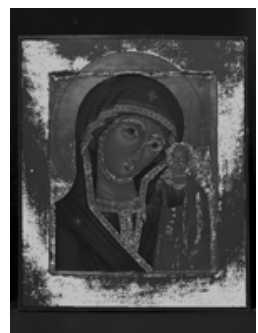

(b) Icon No.2

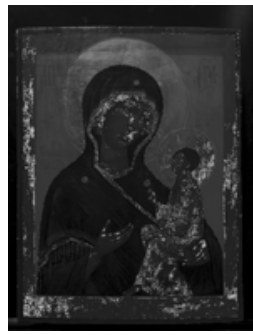

(e) Icon No.6

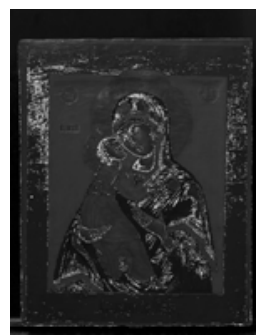

(c) Icon No.3

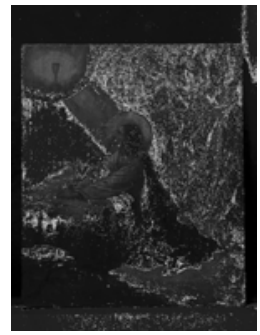

(f) Icon No.7

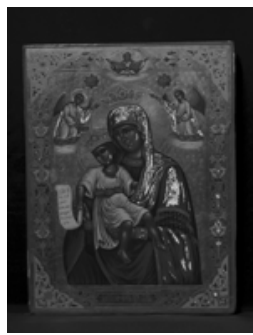

(g) Icon No.8

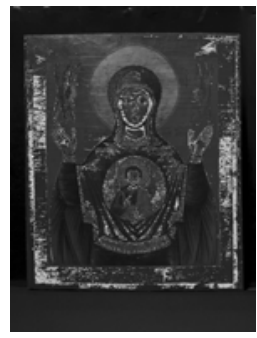

(h) Icon No.9

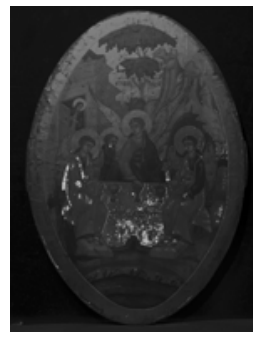

(i) Icon No.10

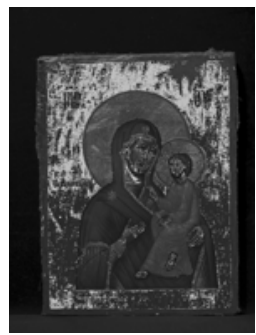

(j) Icon No.18

Fig. 6. Results of the detection of metameric color area by using Method 1

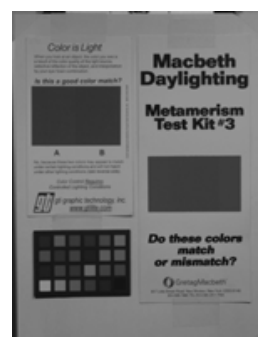

(a) Original chart

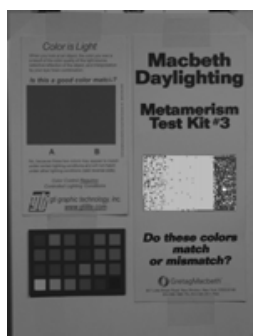

(b) Detected pixels $\left(T_{1}=T_{2}=T_{3}=T_{4}=10.0\right)$

Fig. 7. Results of Method 2 tested for the metamerism test chart 


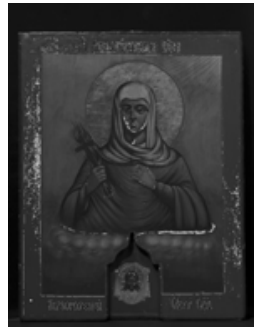

(a) Icon No.1

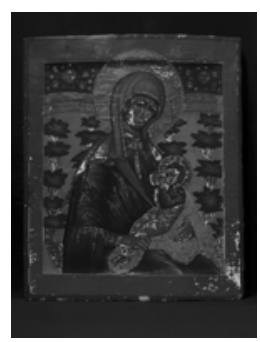

(d) Icon No.4

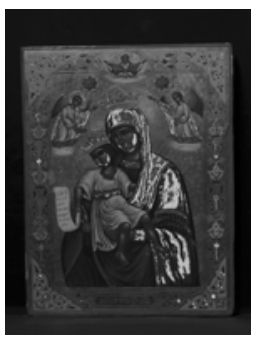

(g) Icon No.8

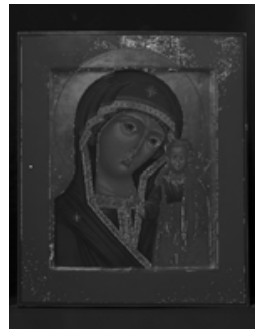

(b) Icon No.2

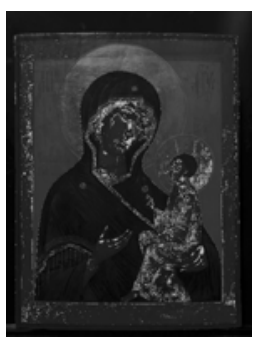

(e) Icon No.6

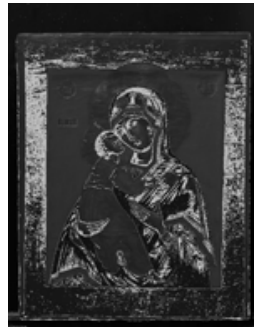

(c) Icon No.3

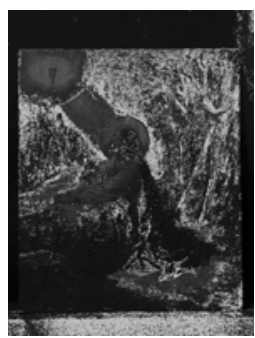

(f) Icon No.7

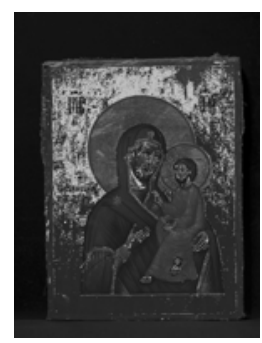

(j) Icon No.18

Fig. 8. Results of the detection of metameric color areas $\left(T_{1}=T_{2}=T_{3}=T_{4}=10.0\right)$

\section{Discussions}

In both methods, a reference pixel is set first, and then metameric colors to the reference pixel are searched by pixel-wise procedure. A reference pixel is set in each Icon, and its position $\left(x_{r}, y_{r}\right)$ remains constant for Method 1 and Method 2. The reference pixel can be set arbitrary in the image, but only one position is used in this study to show the basic performance of the proposed methods.

In the comparison of the detection results, there are no significant differences between Method 1 and Method 2 for the metamerism chart as shown in Figure 5 (d) and Figure 7 (b). However, there are remarkable distinctions in the Icon images as shown in Figure 6 and 8. One of the reasons is that the test chart has a flat surface with the application of homogenous mixture of pigments on the colored areas, while the authentic Icons have uneven surfaces with the use of complicated mixtures of color pigments. Surface reflection property and color mixture model are necessary to en- 
hance accuracy of the detection. Furthermore, methods with scientific analyses are required to conclude whether the detected metameric areas are correct.

\section{Conclusions}

In this study, two kinds of methods are proposed and tested for a test chart and authentic Icons to detect metameric color areas. The proposed methods demonstrate sufficient performance for the test chart, but further improvements are necessary for the experiments on the authentic Icons. The evaluations are discussed as follows:

(1) Consideration of the density fluctuation and mixture of different pigments used in the painting of the Icons.

(2) Consideration of appropriate light sources in the calculation of the tristimulus value.

(3) Comparison between the experimental results from the proposed methods and the results of scientific analyses are necessary to conclude whether the detection by the proposed methods is reliable.

(4) Development of combined investigation methods of measured and estimated spectral reflectance are required for more accurate investigation of materials.

\section{References}

1. Hardeberg, J. Y., Schmitt, F., Brettel, H. H., Crettez, J., Maître, H.: Spectral Imaging in Multimedia, Proc. CIM'98 Colour Imaging in Multimedia (1998) 75-89

2. König, F., Praefcke, W.: A Multispectral Scanner, Proc. CIM'98 Color Imaging in Multimedia (1998) 63-73

3. König, F., Praefcke, W.: Practice of Multispectral Image acquisition, Proc. Electronic Imaging: Processing, Printing and Publishing in Color, SPIE 3409 (1998) 34-41

4. Wyszecki, G., Stiles, W. S.: Color Science 2nd Edition (1982) 180

5. Laamanen, H., Jaaskelainen, T., Hauta-Kasari, M., Parkkinen, J., Miyata, K.: Imaging Spectrograph Based Spectral Imaging System, CGIV 2004 -- Second European Conference on Color in Graphics, Imaging and Vision (2004) 427-430

6. Tsumura, N., Sato, H., Hasegawa, T., Haneishi, H., Miyake, Y.: Limitation of color samples for spectral estimation from sensor responses in fine art painting, Optical Review 6 (1999) 57-61

7. Nishibori, M., Tsumura, N., Miyake, Y.: Why Multispectral Imaging in Medicine?, Journal of Imaging Science and Technology 48 (2004) 125-129

8. Tsumura, $\mathrm{N}$ et al: Estimation of spectral reflectance from multi-band images by multiple regression analysis, Japanese Journal of Optics 27 (1998) 384-391 (in Japanese) 